\title{
CORRECTION
}

\section{Correction to: Treatment and mortality of Klebslella pneumoniae infections in critically ill patients: should we do and predict them better?}

Matteo Bassetti ${ }^{*} \mathbb{D}$, Mura Akova$^{2}$ and Mario Tumbarello ${ }^{3}$

(C) 2018 Springer-Verlag GmbH Germany, part of Springer Nature and ESICM

\section{Correction to: Intensive Care Med https://doi.org/10.1007/s00134-018- 5390-7}

The original version of this article unfortunately contained two mistakes.

The spelling of the name of Dr. Mario Tumbarello was incorrect.

In addition an entry was missing in the reference list. The missing reference is Intensive Care Med. 2018 Oct; 44(10):1709-1719. https://doi.org/10.1007/s0013 4-018-5360-0.

The original article has been corrected.

\section{Author details}

1 Infectious Diseases Clinic, Department of Medicine, University of Udine and Azienda Sanitaria Universitaria di Udine, Piazzale Santa Maria della Misericordia 15, 33100 Udine, Italy. ${ }^{2}$ Department of Infectious Diseases and Clinical Microbiology, School of Medicine, Hacettepe University, Ankara, Turkey. ${ }^{3}$ UOC Malattie Infettive, Fondazione Policlinico Universitario A. Gemelli IRCCS-Istituto Malattie Infettive Università Cattolica del Sacro Cuore, Rome, Italy.

Published online: 9 November 2018

*Correspondence: matteo.bassetti@asuiud.sanita.fvg.it

1 Infectious Diseases Clinic, Department of Medicine, University of Udine and Azienda Sanitaria Universitaria di Udine, Piazzale Santa Maria della Misericordia 15, 33100 Udine, Italy

Full author information is available at the end of the article

The original article can be found online at https://doi.org/10.1007/s0013 4-018-5390-7.

\section{References}

GiViTl Steering Committee, Bertolini G, Nattino G, Tascini C et al (2018) Mortality attributable to different Klebsiella susceptibility patterns and to the coverage of empirical antibiotic therapy: a cohort study on patients admitted to the ICU with infection. Intensive Care Med 44(10):1709-1719. https://doi.org/10.1007/s00134-018-5360-0

\section{Springer}

